Ann. Sci. forest., 1979, 36 (3), 239-262.

\title{
Croissance et nutrition de semis d'un an de hêtre (Fagus silvatica L.) de différentes provenances, élevés sur substratum naturel acide et sur même substratum calcarifié
}

\author{
B. LEPOUTRE et E. TEISSIER du CROS * \\ Professeur de Pédologie ef de Reboisement à l'Ecole Nationale des Ingénieurs \\ des Travaux des Eaux ef Forêts, Domaine des Barres, 45290 Nogent sur Vernisson \\ * Chargé de Recherches, Station d'Amélioration des Arbres Forestiers, I.N.R.A., \\ Centre de Recherches Forestières d'Orléans, Ardon, 45160 Olivet
}

\begin{abstract}
Résumé
La question est posée de savoir si la présence de calcaire ou non dans un sol peut exercer sur le Hêtre une certaine pression de sélection au point que des transferts de provenances de sol acide en sol calcaire, ou vice versa, risquent de compromettre le développement normal des plants.

Des faînes de trois provenances de sols acides d'origines variées et de trois provenances de sols ou de roche-mère calcaires du nord-est de la France ont été mises en prétraitement puis semées en sols contrôlés en pépinière. Les substratum naturels utilisés pour l'élevage des semis, l'un acide, l'autre alcalin, obtenu à partir du premier par addition de carbonate de calcium, ne diffèrent l'un de l'autre que par la teneur en calcaire, donc par le $\mathrm{pH}$.

Le bilan, en fin de première année, montre, qu'à ce stade, le Hêtre n'est pas indifférent à la teneur en calcaire du sol. On observe un comportement variable selon les provenances. Celles qui viennent de sols acides accusent une croissance et un développement moindre en sol calcaire qu'en sol acide. Ceci est la conséquence d'une assimilation minérale, appréciée par analyse foliaire, en déséquilibre. En revanche, des provenances calcaires élevées en milieu acide semblent accuser moins ce transfert. Le sol calcaire semble toxique pour du Hêtre d'origine acide. Le sol d'origine des provenances peut donc avoir exercé sur l'espèce une pression de sélection.

Cette étude sera poursuivie en pépinière après repiquage, puis en forêt.
\end{abstract}

\section{1. - Introduction}

Le hêtre couvre en France 1700000 ha. La variété des sites qu'il occupe est grande : hêtraies de plaine, hêtraies d'altitude, climats océaniques, climats continentaux. S'il semble lié à un état hygrométrique élevé, il ne paraît pas tributaire de la nature du sol. On le rencontre en effet aussi bien sur des sols acides de types variés que sur des sols calciques ou même à calcaire actif. On constate donc chez cette espèce une grande variabilité. Cette variabilité observée (phénotypique) est la résultante d'effets génétiques, d'effets du milieu et de leur interaction. Des sélections de peuplements classés ont été réalisées. Des transferts d'une région à l'autre sont envisagés (Centre Technique du Génie Rural des Eaux et des Forêts, 1977). Il est important de connaître les limites des possibilités de ces transferts. 
Chez d'autres espèces forestières comme l'Epicéa, on sait maintenant que des provenances vosgiennes d'altitude descendues en plaine débourrent tôt et sont sensibles aux gelées tardives (facteur climatique). On sait aussi qu'il est catastrophique d'introduire le Mélèze du Briançonnais en Bretagne pour des raisons pathologiques (facteur biologique). Mais, que sait-on de l'influence des facteurs édaphiques dans les transferts d'espèces à l'intérieur de leur aire naturelle? L'aire du hêtre, dans certaines régions de France, présente l'intérêt d'être continue quel que soit le type du sol. On rencontre ainsi, relativement proches les uns des autres, des peuplements poussant sur sols typiquement calciques ou calcaires et des peuplements sur sols acides. C'est le cas notamment en Lorraine et dans les piémonts vosgiens. Or, les exploitants et les utilisateurs disent préférer le hêtre sur sol calcaire pour sa meilleure qualité (densité plus faible notamment) par rapport au hêtre sur sol acide considéré comme nerveux (Centre Technique du Bois, 1968).

Cette différence de qualité, difficile à retrouver par les analyses densitométriques ( $\left.{ }^{1}\right)$, pourrait être due uniquement ou en partie au milieu. Dans ce cas n'importe quelle provenance locale, d'origine «calcaire » ou non, pourrait donner sur sol calcique le bois de la meilleure qualité. Mais cette différence est vraisemblablement en partie sous dépendance génétique aussi. Dans ce cas, ces « écotypes édaphiques » pourraient conserver tout ou partie de leurs caractéristiques après transfert sur un sol différent, s'ils le supportent.

De nombreux chercheurs ont étudié l'influence du milieu sur la pression de sélection des espèces forestières. Les facteurs du milieu pris en compte sont le plus souvent le climat, l'altitude, la latitude. Rares sont les informations concernant l'influence du sol sur cette pression de sélection.

\section{2. - Les travaux antérieurs}

L'étude des effets respectifs du sol et de l'hérédité a été abordée expérimentalement par quelques chercheurs, surtout sur les résineux (Garbaye et Le Tacon, 1974). II apparaît que selon les espèces et les provenances, il y a en général un $\mathrm{pH}$ optimum de développement. II semble donc utile de s'appliquer à une définition édaphique des provenances.

En matière de feuillus, les peupliers ont été étudiés, mais en général au niveau d'un très faible nombre de clones (Garbaye et Le Tacon op. cit.) voire d'un seul (Garbaye, 1974). Les peupliers noirs de grande culture semblent avoir des aptitudes neutrophiles alors que chez Populus trichocarpa le clone « Fritzi Pauley » paraît sensible au calcaire.

Pour le hêtre, la meilleure information vient d'un essai non publié de Polge, Keller et Thiercelin intitulé : «Effets du sol et de l'hérédité sur la croissance et les caractéristiques anatomiques de jeunes plants de hêtre » (1972). Cet essai comportait deux provenances de sol acide : Epinal et Villers-Cotterêts et une provenance de sol calcaire : Ligny-en-Barrois ; et deux milieux, l'un acide, l'autre calcaire. En matière de croissance, il a été montré que l'effet milieu était prépondérant sur l'effet provenance. Les trois provenances, quelle que fut leur origine, avaient une meilleure crois- 
sance sur sol calcaire. En matière de qualité du bois, par contre, l'effet provenance dominait. Malheureusement, cet essai, bien que très intéressant, comportait des biais à l'origine :

- le milieu acide était constitué d'un horizon $B$ carencé en azote alors que le milieu calcaire était un horizon $A_{1}$ bien équilibré. Leur structure granulométrique était très différente ; sol acide : 70 p. 100 de sable fin et grossier ; sol calcaire : plus de 90 p. 100 d'argile et limons ; ce qui donnait des capacités de rétention en eau très différentes ;

- les plants étaient originaires d'une pépinière (plants $2+1$ ) pour VillersCotterêts ou d'une régénération naturelle (semis de 7 à 8 ans) pour les deux autres provenances.

Tout cela méritait d'être repris en tenant compte des enseignements du premier essai.

$$
\text { * * } *
$$

Les résultats relatés ici proviennent d'observations réalisées à l'issue de la première année d'élevage de semis de hêtre, dans deux milieux, l'un acide, l'autre calcarifié. Ces semis venaient de graines récoltées dans six provenances différentes.

\section{3. - Matériel végétal}

Les six provenances de hêtre choisies, et les informations les concernant sont données au tableau 1.

TABLEAU 1

Localisation des provenances de hêtre Location of beech provenances

\begin{tabular}{|c|c|c|c|c|c|c|c|}
\hline Nom & Abréviation & $\begin{array}{c}\text { Propriétaire } \\
\text { (peuplement } \\
\text { classé) }\end{array}$ & Parcelle & Département & $\begin{array}{l}\text { Latitude } \\
\text { (N) }\end{array}$ & $\begin{array}{l}\text { Longitude } \\
\text { (E) }\end{array}$ & $\begin{array}{c}\text { Altitude } \\
(\mathrm{m})\end{array}$ \\
\hline loinville ... & JOIN & $\begin{array}{c}\text { Forêt communale } \\
(04 \mathrm{HC} \mathrm{05)}\end{array}$ & 34 & Haute Marne & $48^{\circ} 26^{\prime}$ & $5^{\circ} 06^{\prime}$ & 330 \\
\hline $\begin{array}{l}\text { Jrand Verneuil... } \\
\text { Jetite Pierre } \ldots \ldots \\
\text { Sharmes....... } \\
\text { Jarney } . . . \ldots \ldots\end{array}$ & $\begin{array}{l}\text { GRVE } \\
\text { PEPI } \\
\text { CHAR } \\
\text { DARN }\end{array}$ & $\begin{array}{l}\text { Forêt communale } \\
\text { Forêt domaniale } \\
\text { Forêt communale } \\
\text { Forêt domaniale } \\
\quad(09 \mathrm{HC} 05)\end{array}$ & $\begin{array}{r}16 \\
223 \\
18 \\
440\end{array}$ & $\begin{array}{l}\text { Meuse } \\
\text { Bas-Rhin } \\
\text { Vosges } \\
\text { Vosges }\end{array}$ & $\begin{array}{l}49^{\circ} 32^{\prime} \\
48^{\circ} 52^{\prime} \\
48^{\circ} 16^{\prime} \\
48^{\circ} 03^{\prime}\end{array}$ & $\begin{array}{ll}5^{\circ} & 26^{\prime} \\
7^{\prime} & 16^{\prime} \\
5^{\circ} & 04^{\prime} \\
6^{\circ} & 06^{\prime}\end{array}$ & $\begin{array}{l}340 \\
330 \\
350 \\
430\end{array}$ \\
\hline Zhâtillon & CHAT & $\begin{array}{c}\text { Forêt domaniale } \\
\quad(04 \mathrm{HC} 11)\end{array}$ & 458 & Côte-d'Or & $47^{\circ} 47^{\prime}$ & $4^{\circ} 42^{\prime}$ & 380 \\
\hline
\end{tabular}

Ce choix repose sur un gradient climatique et altitudinal assez faibles mais sur des types de sols aussi variés que possible (voir tabl. 2) ( ${ }^{1}$ ).

( $\left.{ }^{1}\right)$ Nous remercions l'Office National des Forêts d'avoir mis à notre disposition des fosses spacieuses et de nous avoir renseigné sur l'état et l'aménagement des peuplements étudiés. 
TABLEAU 2

Caractérisation des stations où les faines ont été récoltées (1)

Description of locations where beechnuts were collected (1)

\begin{tabular}{|c|c|c|c|}
\hline Nom & Type de peuplement & Description pédologique & Observations \\
\hline $\begin{array}{l}\text { JOIN } \ldots \\
\text { GRVE } \ldots\end{array}$ & $\begin{array}{l}\text { TSF en conversion } \\
\text { TSF vieilli }\end{array}$ & $\begin{array}{l}\text { Rendzine sur calcaire marneux } \\
\text { Sol brun calcique sur limon de décar- } \\
\text { bonation }\end{array}$ & $\begin{array}{l}\text { Typiquement calcaire mais }\left({ }^{2}\right) \\
\text { pH } 6 \text { a } 7\end{array}$ \\
\hline PEPI $\ldots \ldots$ & Futaie & $\begin{array}{l}\text { Sol brun acide sableux sur grès vos- } \\
\text { gien }\end{array}$ & \\
\hline $\begin{array}{l}\text { CHAR } \ldots \\
\text { DARN } \ldots\end{array}$ & $\begin{array}{l}\text { Futaie } \\
\text { Futaie }\end{array}$ & $\begin{array}{l}\text { Sol hydromorphe à "pseudogley " } \\
\text { Sol lessivé limoneux à limono-argi- } \\
\text { leux }\end{array}$ & $\begin{array}{l}\mathrm{pH} 4,5 \text { à } 5 \\
\mathrm{pH} 4,2 \text { à } 4,8\end{array}$ \\
\hline CHAT .... & & $\begin{array}{l}\text { Sol brun calcique sur calcaire cryo- } \\
\text { turbé reposant sur calcaire oolothi- } \\
\text { que }\end{array}$ & Typiquement calcaire \\
\hline
\end{tabular}

(1) Cefte caractérisation fait appel à des descriptions floristiques selon Le Tacon ef Timbal (1973) et à l'analyse d'une fosse pédologique par peuplement.

$\left({ }^{2}\right)$ Une partie de cette parcelle, à $150 \mathrm{~m}$ du lieu étudié, est sur un limon légèrement acide. Une partie des faînes, ou même simplement du pollen, a pu venir de cette zone.

Les faines ont été récoltées par l'O.N.F. à l'occasion des faînées de 1974 et 1976. Dès leur arrivée au C.N.R. F., elles ont été séchées puis conservées à $-5^{\circ} \mathrm{C}$.

\section{4. - Méthodologie}

Polge et ses collègues, dans leur étude, étaient partis de plants élevés en pépinière (âge $2+1$ ) ou prélevés en forêt (âge 7 à 8 ans); conscients de ce biais au départ - âge et mise en réserve d'éléments minéraux en conditions différentes et non contrôlées - ils ont été prudents dans l'interprétation des résultats.

Désirant minimiser ces biais, nous sommes partis de deux mille faînes par modalité dont le prétraitement, période d'absorption intense d'eau et d'éléments solubles, a débuté dans le même substrat que l'élevage proprement dit.

Deux milieux d'élevage ont été utilisés :

- milieu acide $(A): 2 / 3$ horizon $A_{1}$ broyé de Bezange

$1 / 3$ terre de bruyère demi-sableuse $\} \mathrm{pH} 4,9\left({ }^{3}\right)$

- milieu calcaire (C) : même substrat que le précédent additionné de 5 p. 100 de $\mathrm{CaCO}_{3}$ magnésien pulvérulent

$\mathrm{pH} 7,5$ à 8.

On peut donc considérer leurs textures comme très proches. La teneur en calcaire et en magnésium étant le seul facteur de variation. Du point de vue chimique, les milieux se sont révélés relativement stables, puisqu'à l'issue de la première année d'élevage, leurs pH étaient encore respectivement de 5,7 et 7,9 (voir tabl. 3).

(3) Nous remercions M. Clément, Chef du Laboratoire de la Station de Recherches sur les Sols Forestiers ef la Fertilisation, pour les mesures de $\mathrm{pH}$ qu'il a faites pour nous. 
TABLEAU 3

Analyse pédologique des milieux d'élevage des semis

Pedological analysis of the seedling culture substrates

Caractères analysés

Milieux

Calcaire Acide

Granulométrie (p. 1000 )

Particules comprises entre 0,000 et $0,002 \mathrm{~mm}$

0,002 et $0,020 \mathrm{~mm} \ldots \ldots \ldots$

0,020 et $0,050 \mathrm{~mm} \ldots \ldots \ldots \ldots \ldots \ldots$

0,050 et $0,200 \mathrm{~mm} \ldots \ldots \ldots \ldots \ldots \ldots$

0,200 et $2,000 \mathrm{~mm}$

$\begin{array}{rr}116 & 102 \\ 61 & 49 \\ 49 & 49 \\ 700 & 734 \\ 74 & 66\end{array}$

Azote « Kjeldahl »

p. $1000 \ldots \ldots \ldots \ldots \ldots \ldots \ldots \ldots$

$\mathrm{pH}$ «Eau ».

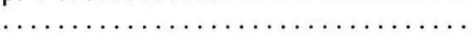

Calcium total H.F. $\left(^{*}\right)$

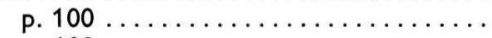

p. $100 \ldots \ldots \ldots \ldots \ldots \ldots \ldots \ldots \ldots$

Magnésium total H.F.

Potassium total H.F.

p. $100 \ldots \ldots \ldots \ldots \ldots \ldots \ldots \ldots \ldots$

Cuivre total H.F.

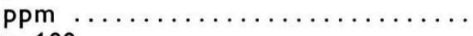

Fer total H.F.

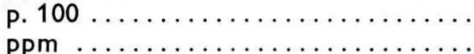

Manganèse total H.F.

p. 100

$\ldots \ldots \ldots \ldots \ldots \ldots \ldots \ldots$

$\begin{array}{cc}2,13 & 1,82 \\ 7,9 & 5,7 \\ 2,49 & 0,36 \\ 0,27 & 0,11 \\ 0,73 & 0,74 \\ 21 & 24 \\ 0,71 & 0,68 \\ 194 & 189 \\ 0,12 & 0,06\end{array}$

* Attaque fluorhydrique.

L'élevage s'est fait en bâches ombrées ; les faînes prétraitées ont été étalées à la surface du substrat (distance : $4 \times 4 \mathrm{~cm}$ ) puis recouvertes d'un centimètre du milieu. Chaque provenance n'était représentée qu'une fois dans chacun des milieux.

\section{5. - Observations et mesures inscrites au programme expérimental}

Cette étude s'est déroulée au Centre National de Recherches Forestières à Nancy en 1977.

- Première semaine de septembre :

- Prélèvement d'une feuille par plant sur deux cents plants pris au hasard dans chaque modalité. Cette feuille fait obligatoirement partie de la paire opposée qui domine les cotylédons.

- Poids frais des douze échantillons de feuilles obtenus (six provenances 2 milieux).

- Poids sec après $3 \mathrm{~h}$ à $120^{\circ} \mathrm{C}$ des mêmes échantillons.

- Longueur et largeur du limbe et produit de ces valeurs pour un échantillon de soixante feuilles par modalité.

- Analyse foliaire de chaque échantillon de deux cents feuilles pour les éléments suivants: N, P, K, Ca, Cu, Mn, Fe, Mg. 
TABLEAU 4

Valeur des caractères mesurés et niveaux de signification des tests statistiques

Values of characteristics measured and significance of statistic tests

\begin{tabular}{|c|c|c|c|c|c|c|c|c|c|c|c|c|c|c|c|}
\hline \multirow{2}{*}{ Provenances } & \multirow{2}{*}{ Milieu } & \multirow{2}{*}{$\begin{array}{l}\text { Survie } \\
\text { p. } 100\end{array}$} & \multirow{2}{*}{$\begin{array}{l}\text { Hauteur } \\
\mathrm{mm}\end{array}$} & \multicolumn{12}{|c|}{ Feuilles } \\
\hline & & & & $\begin{array}{l}\text { Poids } \\
\text { frais } \\
g\end{array}$ & $\begin{array}{l}\text { Poids } \\
\text { sec } \\
g\end{array}$ & $\begin{array}{c}\text { Longueur } \\
\mathrm{mm}\end{array}$ & $\underset{\mathrm{mm}}{\text { Largeur }}$ & $\stackrel{N}{N} 1000$ & $\begin{array}{l}\mathrm{P}_{2} \mathrm{O}_{5} \\
\mathrm{PPm}\end{array}$ & $\begin{array}{c}\mathrm{K} \\
\mathrm{ppm}\end{array}$ & $\begin{array}{c}\mathrm{Ca} \\
\text { p. } 100\end{array}$ & $\begin{array}{c}\mathrm{Cu} \\
\mathrm{Ppm}\end{array}$ & $\begin{array}{l}\mathrm{Mn} \\
\text { Ppm }\end{array}$ & $\begin{array}{c}\mathrm{Fe} \\
\mathrm{Ppm}\end{array}$ & $\begin{array}{l}\mathrm{Mg} \\
\mathrm{Ppm}\end{array}$ \\
\hline $\begin{array}{l}\text { CHAT } \\
\text { JOIN } \\
\text { GRVE } \\
\text { DARN } \\
\text { CHAR } \\
\text { PEPI }\end{array}$ & Acide & $\begin{array}{r}1,7 \\
13,5 \\
20,5 \\
26,5 \\
28,0 \\
13,5\end{array}$ & $\begin{array}{l}108,6 \\
124,7 \\
169,0 \\
140,8 \\
140,4 \\
140,7\end{array}$ & $\begin{array}{l}25,5 \\
27,4 \\
40,5 \\
36,3 \\
38,0 \\
37,3\end{array}$ & $\begin{array}{l}13,1 \\
20,7 \\
18,7 \\
19,4 \\
17,9 \\
17,9\end{array}$ & $\begin{array}{l}44,3 \\
52,9 \\
60,9 \\
60,1 \\
59,9 \\
54,8\end{array}$ & $\begin{array}{l}31,6 \\
36,6 \\
44,3 \\
41,6 \\
43,0 \\
39,6\end{array}$ & $\begin{array}{l}18,80 \\
19,52 \\
19,70 \\
19,70 \\
20,77 \\
19,34\end{array}$ & $\begin{array}{l}2651 \\
2769 \\
2815 \\
2840 \\
2980 \\
2816\end{array}$ & $\begin{array}{l}2546 \\
2725 \\
2801 \\
2710 \\
2845 \\
2529\end{array}$ & $\begin{array}{l}1,73 \\
1,43 \\
1,66 \\
1,62 \\
1,45 \\
1,47\end{array}$ & $\begin{array}{l}21,2 \\
14,1 \\
17,5 \\
14,9 \\
20,0 \\
14,7\end{array}$ & $\begin{array}{rl}1 & 027 \\
& 613 \\
1 & 080 \\
1 & 473 \\
1 & 068 \\
1 & 062\end{array}$ & $\begin{array}{l}595 \\
791 \\
687 \\
712 \\
792 \\
934\end{array}$ & $\begin{array}{ll}2 & 977 \\
3 & 036 \\
3 & 338 \\
3 & 051 \\
2 & 909 \\
3 & 232\end{array}$ \\
\hline $\begin{array}{l}\text { CHAT } \\
\text { JOIN } \\
\text { GRVE } \\
\text { DARN } \\
\text { CHAR } \\
\text { PEPI }\end{array}$ & Calcaire & $\begin{array}{r}12,8 \\
18,5 \\
31,5 \\
8,3 \\
24,5 \\
30,0\end{array}$ & $\begin{array}{l}119,3 \\
138,0 \\
153,5 \\
106,0 \\
123,6 \\
111,9\end{array}$ & $\begin{array}{l}25,9 \\
29,3 \\
31,6 \\
23,1 \\
28,6 \\
25,0\end{array}$ & $\begin{array}{r}13,8 \\
7,8 \\
16,2 \\
12,4 \\
14,1 \\
12,6\end{array}$ & $\begin{array}{l}50,2 \\
51,7 \\
57,7 \\
50,9 \\
54,2 \\
46,3\end{array}$ & $\begin{array}{l}35,8 \\
36,5 \\
42,7 \\
35,8 \\
38,7 \\
34,4\end{array}$ & $\begin{array}{l}18,98 \\
18,72 \\
20,06 \\
18,80 \\
21,13 \\
19,34\end{array}$ & $\begin{array}{l}2580 \\
2793 \\
2723 \\
2444 \\
2722 \\
2623\end{array}$ & $\begin{array}{ll}2 & 438 \\
1 & 962 \\
2 & 375 \\
1 & 532 \\
3 & 048 \\
2 & 103\end{array}$ & $\begin{array}{l}1,65 \\
1,48 \\
1,73 \\
1,74 \\
1,62 \\
1,61\end{array}$ & $\begin{array}{l}11,8 \\
12,7 \\
13,1 \\
13,7 \\
11,8 \\
19,2\end{array}$ & $\begin{array}{r}70 \\
80 \\
182 \\
75 \\
67 \\
61\end{array}$ & $\begin{array}{l}542 \\
461 \\
529 \\
598 \\
561 \\
535\end{array}$ & $\begin{array}{ll}3 & 613 \\
3 & 220 \\
3 & 731 \\
3 & 586 \\
3 & 276 \\
3 & 626\end{array}$ \\
\hline \multirow[t]{2}{*}{ Moyennes } & Acide & 17,3 & 140,8 & 34,2 & 18,0 & 56,5 & 40,2 & 19,64 & 2812 & 2693 & 1,56 & 17,1 & 1054 & 752 & 3091 \\
\hline & Calcaire & 20,9 & 125,4 & 27,3 & 12,8 & 51,8 & 37,3 & 19,51 & 2648 & 2243 & 1,64 & 13,7 & 89 & 538 & 3509 \\
\hline $\begin{array}{c}\text { Effet } \\
\text { provenance }\end{array}$ & $\begin{array}{c}\text { Acide } \\
\text { Calcaire }\end{array}$ & $\begin{array}{l}1 \\
1\end{array}$ & $\begin{array}{l}* * \\
* *\end{array}$ & $\begin{array}{l}1 \\
1\end{array}$ & $\begin{array}{l}1 \\
1\end{array}$ & $\begin{array}{l}* * \\
* *\end{array}$ & $\begin{array}{l}* * \\
* *\end{array}$ & $\begin{array}{l}1 \\
1\end{array}$ & $\begin{array}{l}1 \\
1\end{array}$ & $\begin{array}{l}1 \\
1\end{array}$ & $\begin{array}{l}1 \\
1\end{array}$ & $\begin{array}{l}1 \\
1\end{array}$ & $\begin{array}{l}1 \\
1\end{array}$ & $\begin{array}{l}1 \\
1\end{array}$ & $\begin{array}{l}i \\
1\end{array}$ \\
\hline \multicolumn{2}{|c|}{ Effet milieu } & NS & NS & NS & * & NS & NS & NS & * & NS & NS & NS & $* *$ & $* *$ & $* *$ \\
\hline \multicolumn{2}{|c|}{ Interaction } & 1 & $* *$ & 1 & 1 & $* *$ & $* *$ & 1 & 1 & 1 & 1 & 1 & 1 & 1 & 1 \\
\hline
\end{tabular}


- Hiver.

- Hauteur totale d'un échantillon de soixante plants par modalité.

- Analyse pédologique des milieux d'élevage.

\section{6. - Résultats}

L'ensemble des résultats et le niveau de signification des analyses statistiques réalisées figurent au tableau 4.

\section{1. - Comportement et morphologie des plants}

6.11. La survie (fig. 1).

Les valeurs observées intègrent le pouvoir germinatif des lots de faînes et les effets des attaques parasitaires en cours de croissance.

Les valeurs individuelles des provenances dans chacun des milieux sont très variables. Le pouvoir germinatif de Chatillon et Joinville était certainement bas. Peutêtre les effets de l'attaque de fonte (Rhizoctonia solani) ont-ils été différents selon les

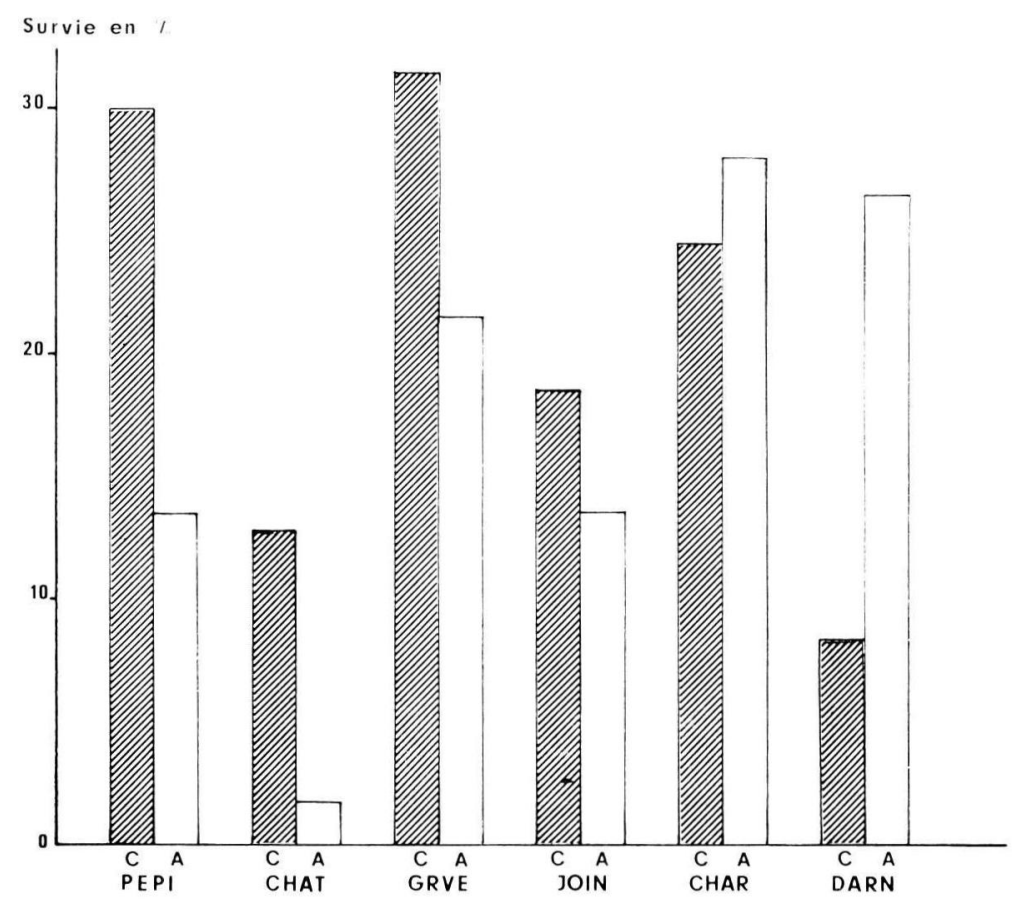

FIG. 1. - Survie, classement des provenances par ordre croissant de A-C.

$A=$ survie en milieu acide.

$\mathrm{C}=$ survie en milieu calcaire.

Survival, classification of provenances for increasing values of A-C.

$A=$ survival on acid substrate.

$\mathrm{C}=$ survival on alcaline substrate. 
provenances? Mais ce qui semble plus important, c'est que, si l'on avait jugé la survie sur la moyenne des provenances on n'aurait pas décelé de différence entre les deux milieux ; en fait, les provenances réagissent de façon très marquée selon les milieux. Trois des quatre provenances, à survie plus élevée en milieu calcaire qu'en milieu acide, viennent de stations « calcaires » ou « neutres ». Petite Pierre fait exception. Par contre Charmes et Darney, toutes deux considérées comme «acides »ont une meilleure survie en milieu acide.

\subsection{La hauteur (fig. 2).}

Ayant réalisé les mesures individuellement sur soixante plants par modalité (trente-trois seulement pour Chatillon en milieu acide), nous avons procédé à une analyse de variance, d'abord dans chacun des milieux, puis globalement dans les deux (facteurs provenances, milieux et interaction). Dans chacun des milieux les provenances montrent une forte variabilité ; ce qui n'a rien d'étonnant puisqu'aucune

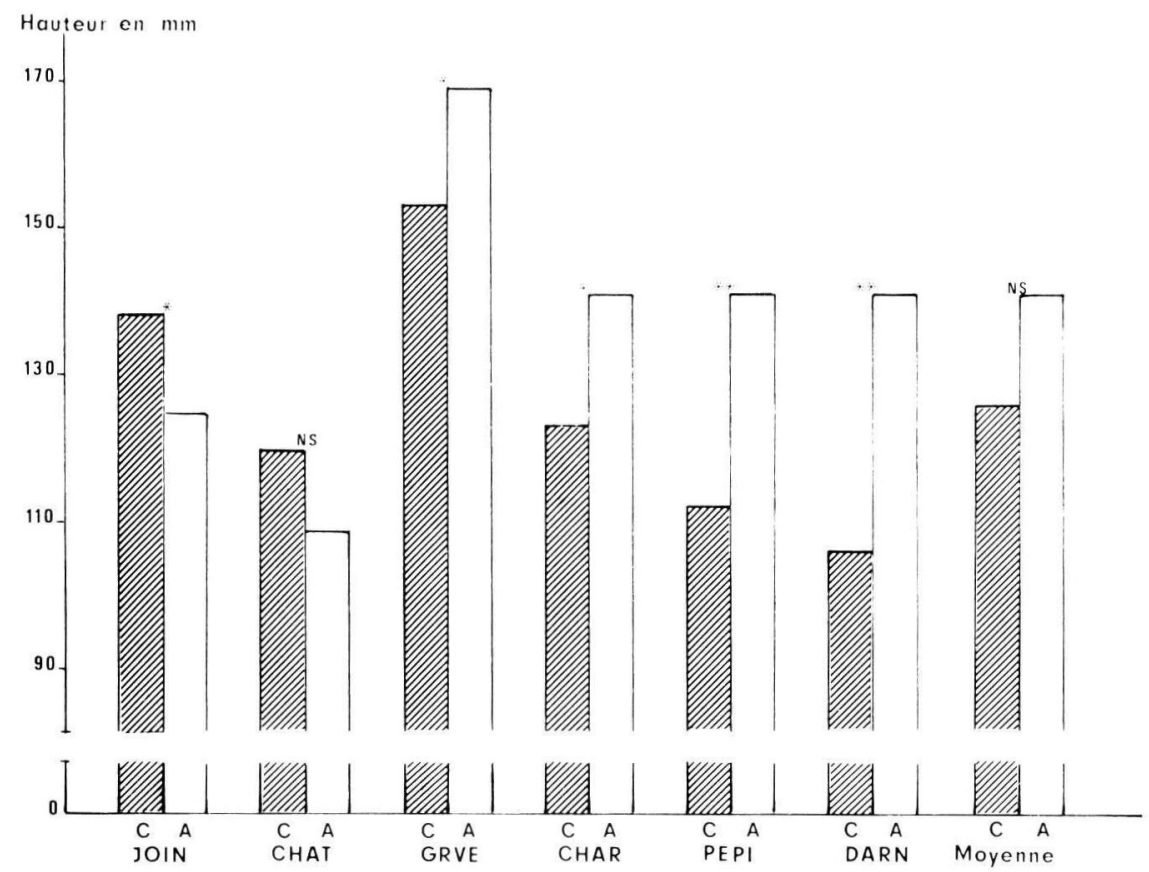

FIG. 2. - Hauteur, classement des provenances par ordre croissant de A-C.

$\mathrm{A}=$ hauteur en milieu acide.

$\mathrm{C}=$ hauteur en milieu calcaire.

* différence significative au seuil de 5 p. 100.

** différence significative au seuil de 1 p. 100.

NS pas de différence significative.

Height, classification of provenances for increasing values of A-C.

$A=$ height on acid substrate.

$C=$ height on alcaline substrate.

* difference significant at 5 p. 100 level.

** difference significant at 1 p. 100 level.

NS no significant difference. 
sélection préalable n'avait été réalisée. L'effet du milieu jugé globalement est nul. Par contre les provenances ont un comportement très différent selon leur origine (interaction). Joinville et Chatillon ont une meilleure croissance en milieu calcaire, or, elles ont toutes deux été considérées comme « calcaire » dans la caractérisation des stations (tabl. 2).

Sur la figure 2, bien que voisine des provenances calcaires, Grand Verneuil ne se comporte pas comme elles, pour la hauteur ; celle-ci est en effet meilleure en milieu acide qu'en milieu calcaire. Ceci ne nous étonne pas puisque le sol de ce peuplement est décarbonaté sur $0,80 \mathrm{~m}$ à $1 \mathrm{~m}$ de profondeur et que son $\mathrm{pH}$ a été estimé aux environs de 6 ou 7. Les autres provenances, enfin, se sont mieux développées en milieu acide. Ce phénomène semble devoir être rapproché de l'origine " acide » de ces trois provenances.

Il importe de souligner ici, que les hauteurs peuvent avoir été influencées par la densité des semis dans les bâches. On remarque en effet que Chatillon, en milieu acide, a un très faible taux de survie. II en est de même pour Darney en milieu calcaire. Or ces deux modalités ont la plus faible croissance en hauteur. Ces plants ne paraissent donc pas avoir profité de cette faible densité pour mieux se développer.

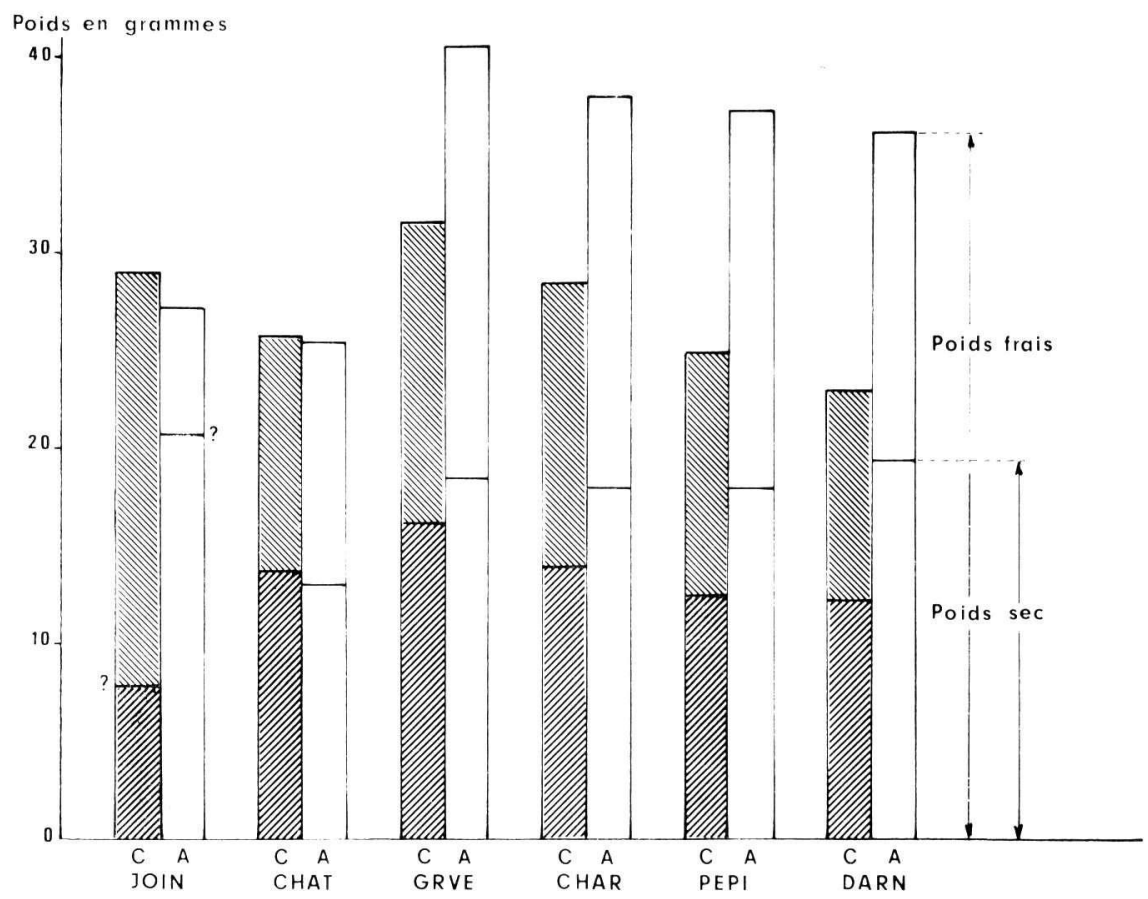

FIG. 3. - Poids des échantillons de feuilles, classement des provenances par ordre croissant de la valeur de A-C pour le poids frais.

$\mathrm{A}=$ poids des feuilles en milieu acide.

$\mathrm{C}=$ poids des feuilles en milieu calcaire.

Leaf sample weights, classification of provenances for increasing values of A-C for fresh weight.

$A=$ leaf weight on acid substrate.

$\mathrm{C}=$ leaf weight on alcaline substrate. 
6.13. Poids des échantillons de feuilles.

Le poids frais des échantillons ne nous apporte pas d'information nouvelle par rapport à la hauteur des plants. D'ailleurs, ces deux caractères sont étroitement liés ( $r=0,91 *$ en milieu acide et $0,95^{* *}$ en milieu calcaire) ; ils conduisent au même classement des provenances (fig. 2 et 3 ).

L'observation du poids sec des échantillons foliaires ne nous est d'aucun recours du fait de la donnée aberrante de Joinville (erreur de pesée ?).

\subsection{Grandeurs des feuilles.}

La longueur et la largeur du limbe sont étroitement corrélées, aussi bien entre moyennes de provenances $(r=0,98 * * *)$ qu'au niveau individuel $(0,67 * * *)$. Par contre, ces valeurs ne sont pas liées avec la hauteur des plants (moyennes de provenances). Aussi nous n'avons retenu comme caractère à analyser, que la longueur du limbe (fig. 4).

Tout comme pour la hauteur, nous disposions de mesures individuelles sur soixante feuilles par provenance (trente pour Chatillon en milieu acide) ce qui nous a permis d'évaluer statistiquement les interactions. Dans les deux milieux, pris indé-

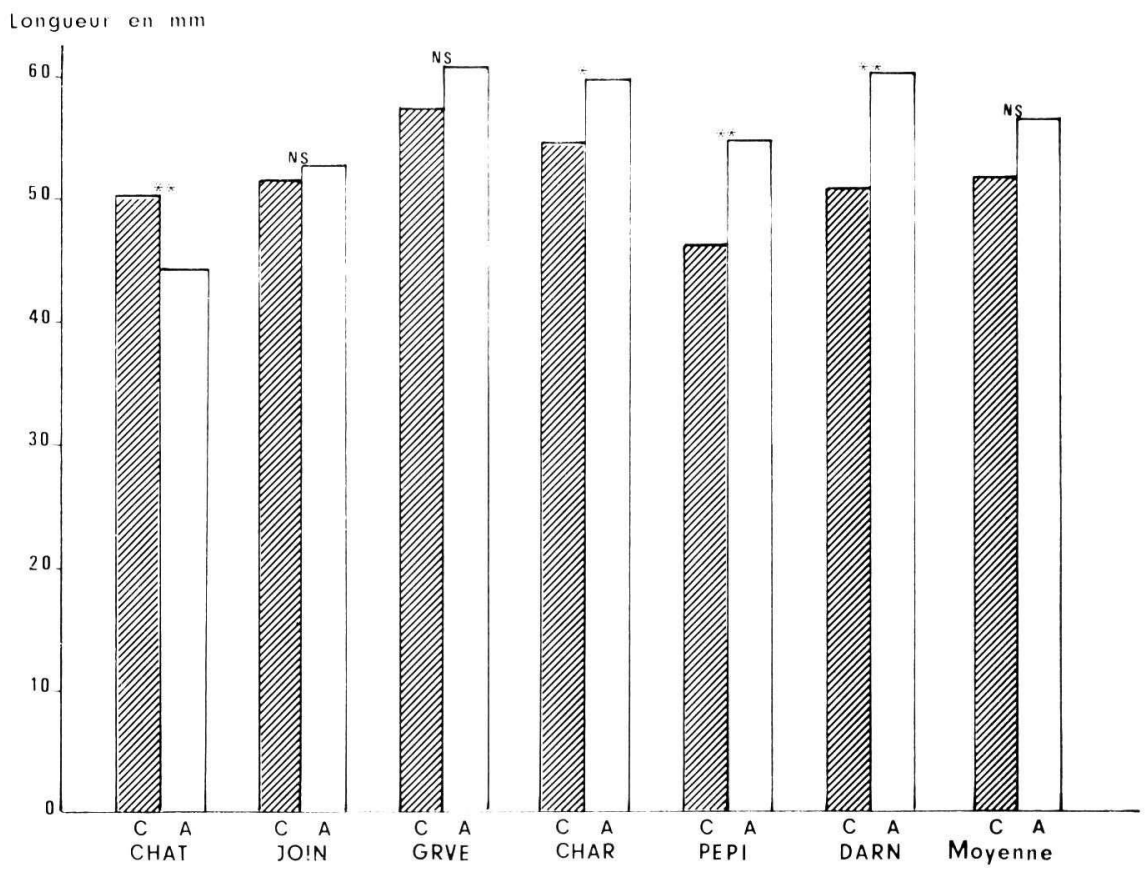

FIG. 4. - Longueur du limbe, classement des provenances par ordre croissant de la valeur de A-C.

$A=$ longueur du limbe en milieu acide.

$\mathrm{C}=$ longueur du limbe en milieu calcaire.

Niveaux des tests statistiques : comme pour la figure 2.

Leaf blade length, classification of provenances for increasing values of A-C.

$A=$ Leaf blade length on acid substrate.

$\mathrm{C}=$ Leaf blade length on alcaline substrate.

Level of statistic tests : see figure 2. 
pendamment, la variabilité est importante. Lorsque l'analyse est faite globalement, la variabilité entre provenances et entre milieux disparaît du fait d'une forte interaction. A gauche sur la figure 4, Chatillon a de plus grandes feuilles en milieu calcaire qu'en milieu acide. Joinville et Grand Verneuil ne montrent pas de différence selon les milieux. Ensuite viennent les provenances «acides» dont les feuilles sont plus développées en milieu acide qu'en milieu calcaire.

On observe fréquemment chez les feuillus que le développement des feuilles est le reflet direct de l'adaptation des plants (cf. des photos de fevilles de peuplier de Fritzi Pauley en conditions favorables; Garbaye, op. cit.). Cette adaptation peut être due à la sortie d'une crise (crise de transplantation, enracinement d'une bouture), elle est certainement ici la démonstration que le hêtre ne se développe pleinement que dans son milieu « habituel ».

Au vu des caractères physiques et morphologiques étudiés jusqu'ici : hauteur des plants, masse foliaire, grandeur des feuilles, deux résultats importants apparaissent :

- le milieu a modelé le hêtre au point que dès la première année de végétation il ne se développe pleinement que dans son milieu habituel, acide ou calcaire, auquel il se serait donc adapté. Il est ainsi probable que le milieu a exercé sur lui une certaine pression de sélection ;

- les provenances d'origine « calcaire » semblent moins souffrir d'un élevage dans un milieu inhabituel (acide) que les provenances d'origine « acide » transférées en sol calcaire.

Or ces plus ou moins grandes difficultés d'adaptation sont le reflet de leur absorption minérale, ainsi que nous allons le voir.

\section{2. - Analyses foliaires}

L'observation des moyennes, à la base du tableau 4, montre que le comportement en milieu calcaire diffère du comportement en milieu acide principalement par une plus grande absorption de calcium, mais aussi du magnésium (le carbonate de calcium utilisé était magnésien) et une chute de l'assimilation minérale. Cette dernière est particulièrement marquée pour le manganèse, le fer et le phosphore et à un moindre degré pour le cuivre et le potassium, bien que le résultat de Charmes en sol calcaire masque un haut degré de signification pour les autres provenances.

La caractérisation des provenances, non plus par la description pédologique des stations, mais par l'analyse foliaire de semis qui en sont issus est difficile.

La difficulté réside dans le fait que la nutrition minérale présente souvent des seuils en-deçà ou au-delà desquels les comportements de l'essence peuvent ne pas être influencés. Par ailleurs en l'absence de seuils, il n'y a pas toujours proportionnalité entre un critère biologique et une teneur en un élément. Enfin des éléments peuvent être assimilés sans profit (consommation de luxe).

Pour ces raisons, nous avons donc cherché à individualiser, des comportements analogues ou très différenciés des provenances, sans jamais essayer de tirer des conclusions sur la signification des valeurs absolues chiffrées. De la sorte, comme dans une analyse de correspondance, nous avons regroupé les provenances dont l'assimilation ou les variations d'assimilation pour un ou plusieurs éléments sont voisines lorsque cela était possible. 
Il paraît nécessaire de bien définir ici la méthode d’interprétation du diagnostic foliaire utilisée.

En effet, plusieurs possibilités d'interprétation s'offrent à nous.

1) Utiliser un classement selon les valeurs croissantes ou décroissantes des taux de l'élément assimilé et confronter ce classement avec celui du comportement.

Cette méthode implique, a priori, une loi régulière croissante ou décroissante entre le comportement et l'assimilation ce qui est en contradiction avec la loi de Steenbjerg. Il est donc peu probable qu'on puisse trouver une telle relation, et à l'inverse s'il en était trouvé une, elle ne pourrait sans doute qu'être fortuite donc ininterprétable.

2) Utiliser un classement faisant apparaître les modifications de chaque provenance lorsqu'on les déplace d'un milieu à un autre. On peut alors penser que toute modification du comportement, s'il en existe une, a des chances d'être le reflet d'une modification parallèle d'un ou plusieurs éléments nutritionnels.

La méthode 2 a été retenue ici puisqu'elle a davantage de chance de faire apparaître les éléments assimilés ayant une influence sur le comportement, ou également, les provenances sensibles aux variations d'assimilation ou aux variations de milieu (provenances aptes à extraire les éléments minéraux en sol calcaire par exemple).

La démarche a été la suivante : compte tenu de la modification importante de l'assimilation minérale en sol calcaire, on est logiquement amené à étudier les équi-

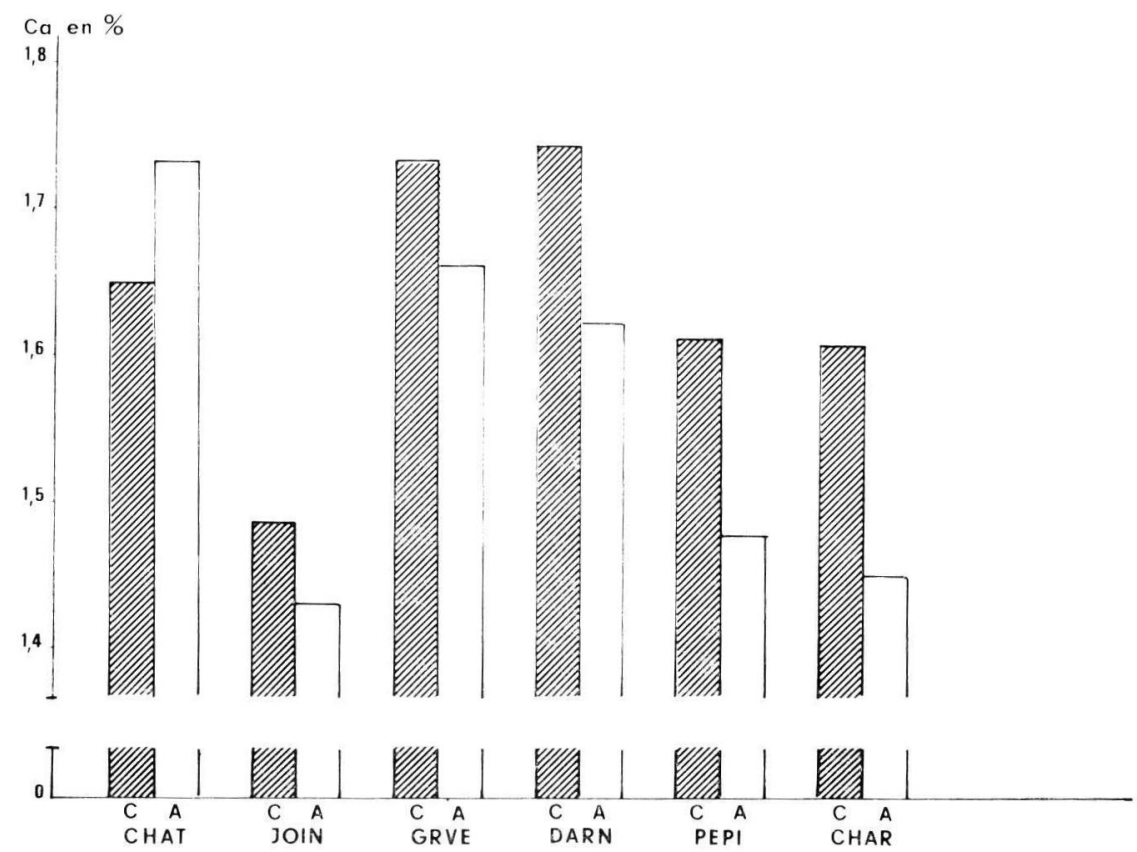

FIG. 5. - Calcium, classement des provenances par ordre croissant de la valeur de C-A.

$\mathrm{A}=$ concentration de $\mathrm{Ca}$ dans les feuilles en milieu acide.

$\mathrm{C}=$ concentration de $\mathrm{Ca}$ dans les feuilles en milieu calcaire.

Calcium, classification of provenances for increasing values of C-A.

$\mathrm{A}=$ concentration of $\mathrm{Ca}$ in leaves on acid substrate.

$\mathrm{C}=$ concentration of $\mathrm{Ca}$ in leaves on alcaline substrate. 
libres entre les cations alcalino-terreux $\left(\mathrm{Ca}^{++}\right.$et $\left.\mathrm{Mg}^{++}\right)$d'une part, les autres cations minéraux et le phosphore, la potasse et l'azote d'autre part.

Mais avant il a paru utile d'analyser l'absorption des cations, en fonction du milieu.

\subsection{Le calcium.}

Comme pour les caractères étudiés jusqu'ici nous avons basé notre observation non pas sur un classement global dans chacun des milieux, mais sur le classement des différences d'assimilation des provenances entre les deux milieux (fig. 5). On voit alors nettement apparaître à gauche les deux provenances « calcaires", puis Grand Verneuil considéré comme «neutre » et à droite les provenances «acides». Ces dernières paraissent donc modifier davantage leur nutrition lorsqu'on les place en sol calcaire, vers une absorption de $\mathrm{Ca}^{++}$plus élevée, qui ne leur est pas habifuelle. A l'inverse les provenances calcaires telles que Chatillon et Joinville modifient moins leur nutrition calcique lorsqu'elles sont placées en sol acide. Cela devrait vraisemblablement modifier l'assimilation de certains autres éléments minéraux.

\subsection{Le magnésium.}

Le magnésium joue ici un rôle parasite (non prévu dans l'expérimentation) du fait de sa présence dans le carbonate de calcium apporté. On observe (fig. 6) une

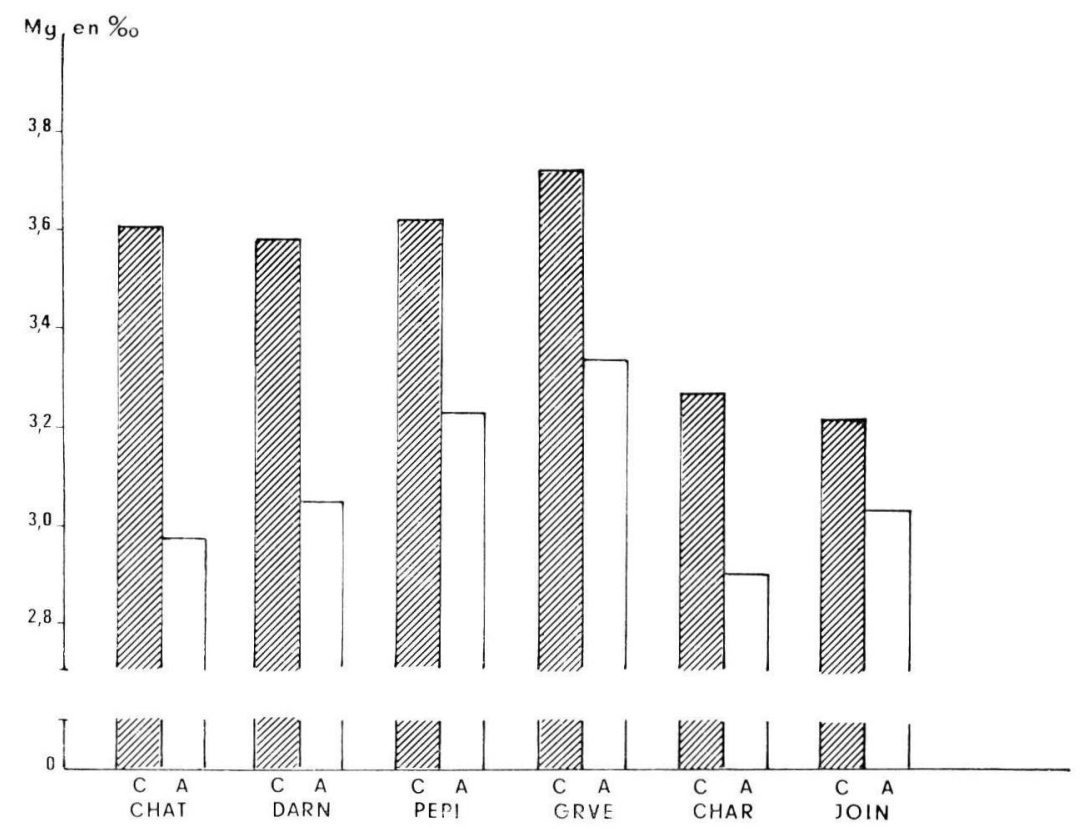

FIG. 6. - Magnésium, classement des provenances par ordre croissant de la valeur de A-C.

$\mathrm{A}=$ concentration de $\mathrm{Mg}$ dans les feuilles en milieu acide.

$\mathrm{C}=$ concentration de $\mathrm{Mg}$ dans les feuilles en milieu alcalin.

Magnesium, classification of provenances for increasing values of A-C.

$\mathrm{A}=$ concentration of $\mathrm{Mg}$ in leaves on acid substrate.

$\mathrm{C}=$ concentration of $\mathrm{Mg}$ in leaves on alcaline substrate. 
absorption relativement plus forte de toutes les provenances en milieu calcaire. Les provenances ne se comportent pas de façon uniforme, mais aucune loi pouvant se rattacher à la nature du substrat d'origine ne semble pouvoir être tirée de ce classement.

\subsection{L'équilibre Ca-Fe.}

Aucune relation $\mathrm{Ca}-\mathrm{Fe}$ ne peut être mise en évidence sur l'ensemble des provenances.

\subsection{L'antagonisme Ca-Fe.}

C'est celui qui caractérise souvent les espèces calcicoles et calcifuges. La figure 7 montre la variation du taux d'assimilation du fer pour chaque provenance, lors de son passage du milieu acide au milieu calcaire, selon la variation du $\mathrm{Ca}^{++}$absorbé.

Le regroupement selon une loi semble difficile. Tout au plus pouvons nous remarquer qu'un groupe de provenances a une assimilation du fer qui varie peu quelle que soit la variation d'absorption du $\mathrm{Ca}^{++}$et que l'autre groupe accuse une variation plus forte de cette teneur en fer. Dans le premier groupe, nous trouvons Chatillon,

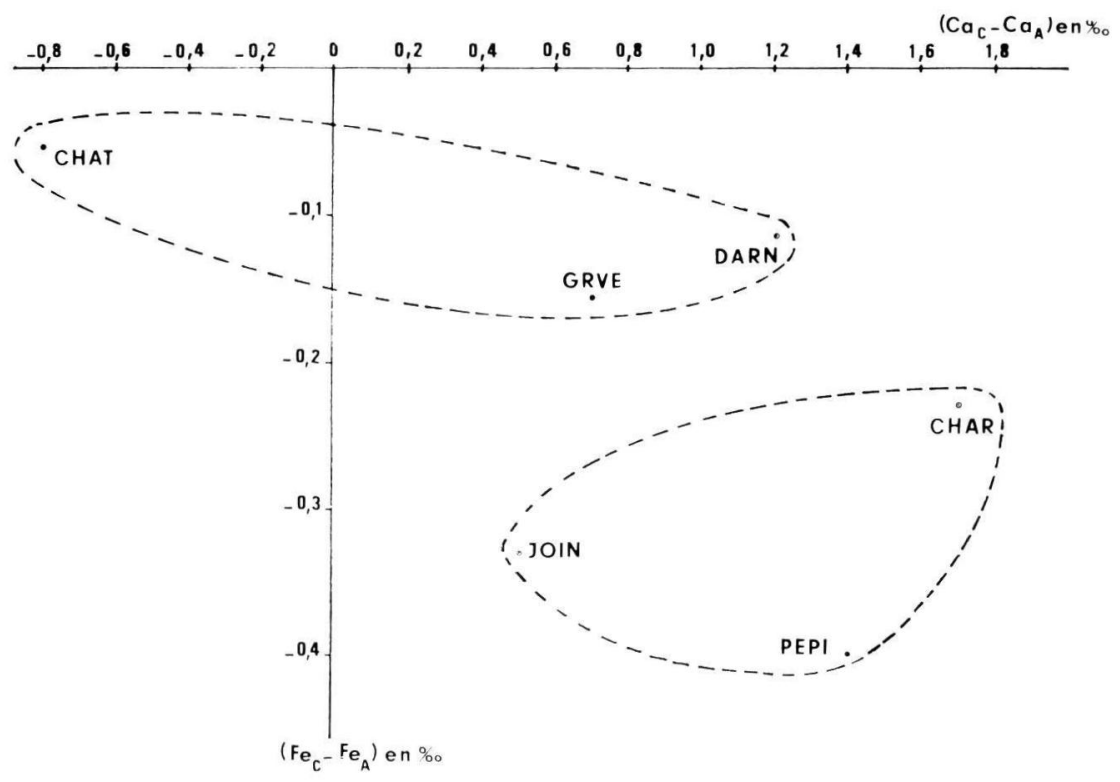

FIG. 7. - Différence d'assimilation du fer en fonction de celle du calcium entre le substratum calcaire et le substratum acide.

$\mathrm{Ca}_{\mathrm{c}}=$ concentration en Ca dans les feuilles en milieu calcaire.

$\mathrm{Ca}_{\mathrm{A}}=$ concentration en $\mathrm{Ca}$ dans les feuilles en milieu acide.

$\mathrm{Fe}_{\mathrm{c}}=$ concentration en Fe dans les feuilles en milieu calcaire.

$\mathrm{Fe}_{\mathrm{A}}=$ concentration en Fe dans les feuilles en milieu acide.

Difference between alcaline and acid substrates for Fe assimilation in connection with Ca assimilation.

$\mathrm{Ca}_{\mathrm{c}}=\mathrm{Ca}$ concentration in leaves on alcaline substrate.

$\mathrm{Ca}_{\mathrm{A}}=\mathrm{Ca}$ concentration in leaves on acid substrate.

$\mathrm{Fe}_{\mathrm{c}}=\mathrm{Fe}$ concentration in leaves on alcaline substrate.

$\mathrm{Fe}_{\mathrm{A}}=\mathrm{Fe}$ concentration in leaves on acid substrate. 
typiquement " calcaire », Grand Verneuil dont le substrat est calcaire et Darney, typiquement " acide \#. Dans le deuxième groupe, Petite Pierre et Charmes sont typiquement « acides». La présence de Joinville dans ce groupe pourrait autoriser quelques doutes dans la définition purement «calcaire» de cette provenance. Cela rejoindrait alors la restriction que nous avions faite au tableau 2 .

\subsection{L'équilibre $\mathrm{Fe}-\mathrm{Mg}$.}

Comme pour le calcium il ne semble pas y avoir de relation $\mathrm{Mg}$-Fe assimilés (teneurs globales) pour l'ensemble des provenances.

\subsection{La synergie Fe-Mg.}

La concentration du magnésium dans le milieu calcaire est supérieure à celle du milieu acide, sans doute du fait de l'emploi d'un carbonate de calcium magnésien. La supériorité globale de l'assimilation du $\mathrm{Mg}$ en milieu calcaire, ne nous surprend donc pas, la figure 8 semble cependant mettre en évidence une synergie Fe-Mg :

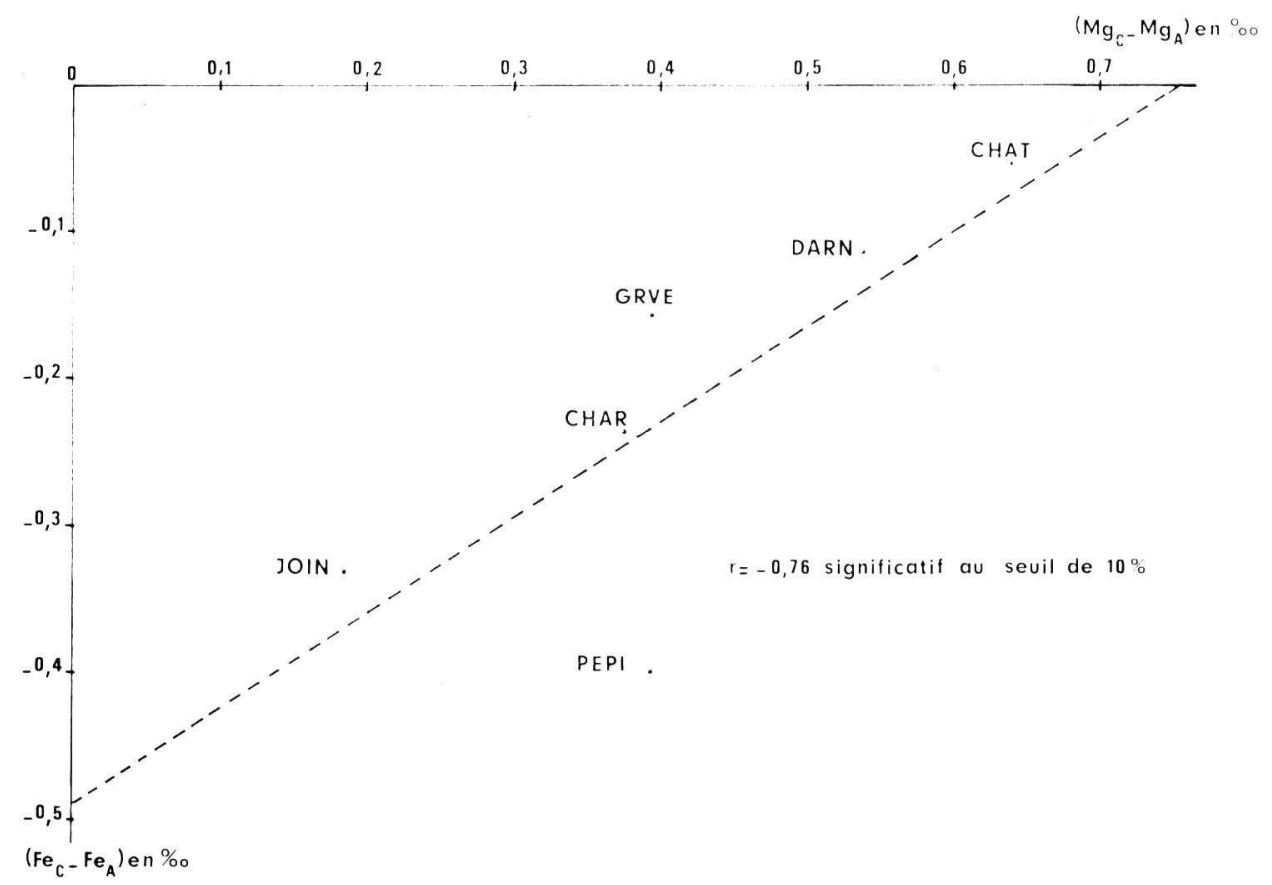

FIG. 8. - Différence d'assimilation du fer en fonction de celle du magnésium entre le substratum calcaire ef le substratum acide.

$\mathrm{Fe}_{\mathrm{c}}=$ concentration en Fe dans les feuilles en milieu calcaire.

$\mathrm{Fe}_{\mathrm{A}}=$ concentration en Fe dans les feuilles en milieu acide.

$\mathrm{Mg}_{\mathrm{c}}=$ concentration en $\mathrm{Mg}$ dans les feuilles en milieu calcaire.

$\mathrm{Mg}_{\mathrm{A}}=$ concentration en $\mathrm{Mg}$ dans les feuilles en milieu acide.

Difference between alcaline and acid substrates for Fe assimilation in connection with $\mathrm{Mg}$ assimilation.

$\mathrm{Fe}_{\mathrm{c}}=\mathrm{Fe}$ concentration in leaves on alcaline substrate.

$\mathrm{Fe}_{\mathrm{A}}=\mathrm{Fe}$ concentration in leaves on acid substrate.

$\mathrm{Mg}_{\mathrm{c}}=\mathrm{Mg}$ concentration in leaves on alcaline substrate.

$M g_{A}=M g$ concentration in leaves acid substrate. 
les provenances dont l'augmentation d'absorption en $\mathrm{Mg}^{++}$est la plus forte voient leur absorption en fer la moins modifiée.

Ceci est assez étonnant. II faut conclure : du fer,

- soit qu'il existe réellement une incidence de l'absorption du $\mathrm{Mg}$ sur celle

- soit que d'autres facteurs liés au Mg sont en cause,

- soit qu'il existe une sensibilité intraspécifique variable selon les provenances, très forte de Joinville et au contraire très faible de Chatillon par exemple.

Cette sensibilité serait alors indépendante du milieu d’origine (cause génétique) ou bien il ne faudrait pas considérer Joinville comme venant d'un milieu calcaire. Dans cette dernière hypothèse les provenances de milieu calcique ou calcaire seraient moins sensibles à la variation du taux de $\mathrm{Mg}$ du milieu (Chatillon, Grand Verneuil) que les provenances acides. Ces doutes ne peuvent être levés pour le moment.

\subsection{Le cuivre (fig. 9).}

L'interprétation de l'effet provenance dans la différence d'assimilation du Cu entre les milieux acide et calcaire n'est pas aisée. Elle fait apparaître une très forte

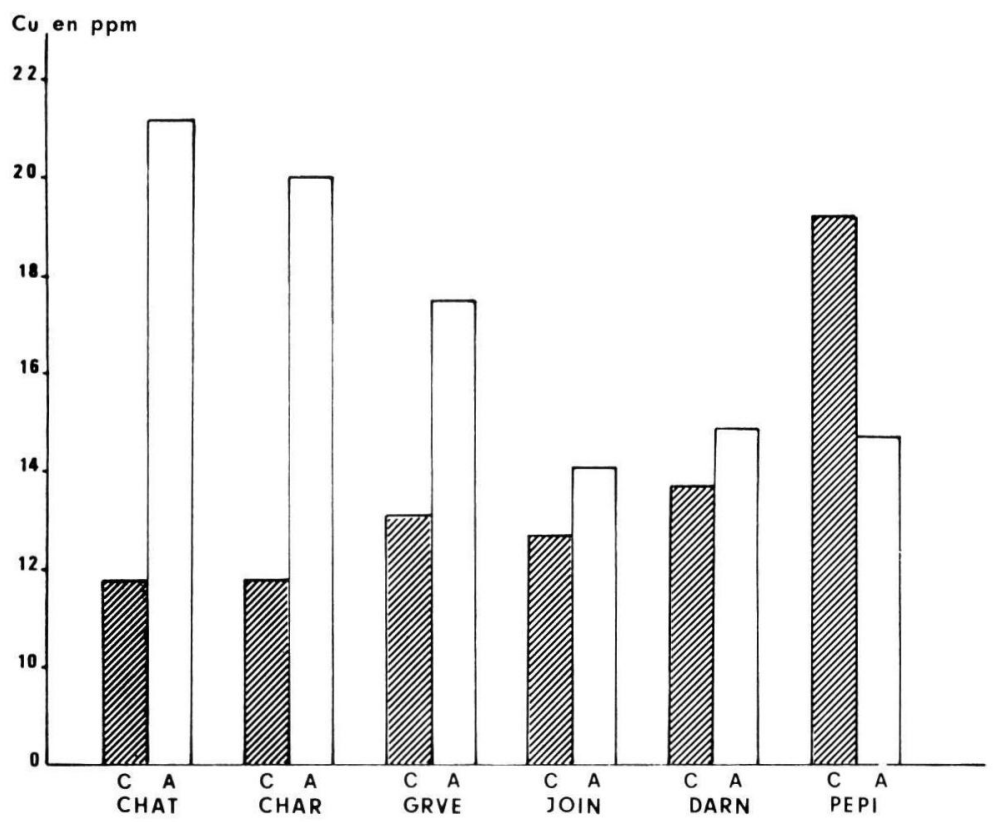

FIG. 9. - Cuivre, classement des provenances par ordre décroissant de la valeur de A-C.

$\mathrm{A}=$ concentration en Cu en milieu acide.

$\mathrm{C}=$ concentration en Cu en milieu calcaire.

Copper, classification of provenances for decreasing values of A-C.

$\mathrm{A}=$ concentration of $\mathrm{Cu}$ in leaves on acid substrate.

$\mathrm{C}=$ concentration of $\mathrm{Cu}$ in leaves on alcaline substrate. 
interaction. On trouve d'abord un groupe de provenances (Chatillon, Charmes et Grand Verneuil) dont l'assimilation du Cu est réduite de plus du quart par la présence de carbonate dans le sol, puis un groupe intermédiaire (Joinville, Darney) dont l'assimilation du cuivre semble indépendante du milieu, et enfin, Petite Pierre qui a absorbé plus de cuivre en milieu calcaire qu'en milieu acide. Il y a donc vraisemblablement indépendance entre la caractérisation « calcaire 》 ou " acide » des provenances et leur aptitude à assimiler le cuivre en milieu acide ou calcaire après transfert. II pourrait y avoir une caractéristique génétique.

\subsection{Le manganèse.}

Le milieu de culture joue un rôle primordial dans l'assimilation de cet élément; en effet le manganèse est insolubilisé en milieu alcalin et n'est donc plus à la disposition des plants. La figure 10 met néanmoins en évidence, malgré une certaine uniformisa-

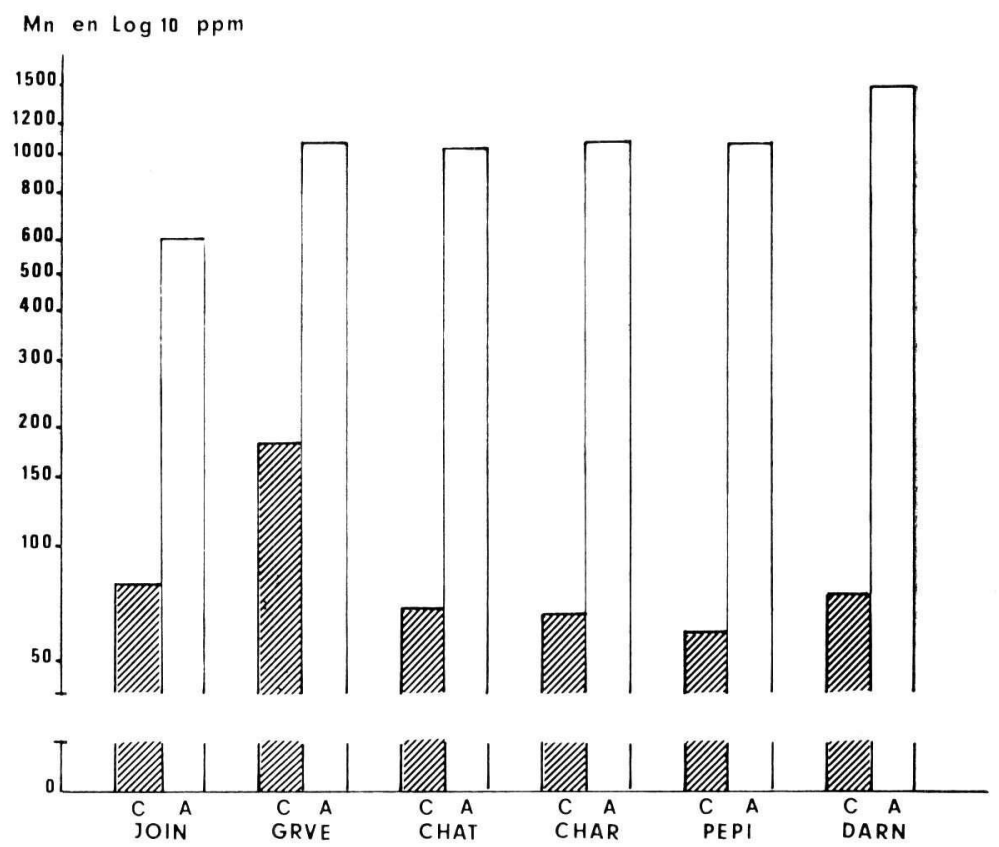

FIG. 10. - Manganèse, classement des provenances par ordre croissant de la valeur de A-C.

$\mathrm{A}=$ concentration en $\mathrm{Mn}$ en milieu acide.

$\mathrm{C}=$ concentration en $\mathrm{Mn}$ en milieu calcaire.

Manganese, classification of provenances for increasing values of A-C.

$A=$ concentration of $\mathrm{Mn}$ in leaves on acid substrate.

$\mathrm{C}=$ concentration of $\mathrm{Mn}$ in leaves on alcaline substrate.

tion des assimilations en milieu alcalin, des provenances qui réussissent à absorber le manganèse moins bien quand le $\mathrm{pH}$ est élevé que lorsqu'il est bas. Ces provenances viennent précisément d'un milieu acide. 
6.29. Le phosphore.

L'assimilation du phosphore par les jeunes hêtres semble être liée à l'aptitude à supporter le calcium. La figure 11 met en évidence un groupe de provenances dont l'assimilation du phosphore est relativement indépendante de celle du calcium, ce

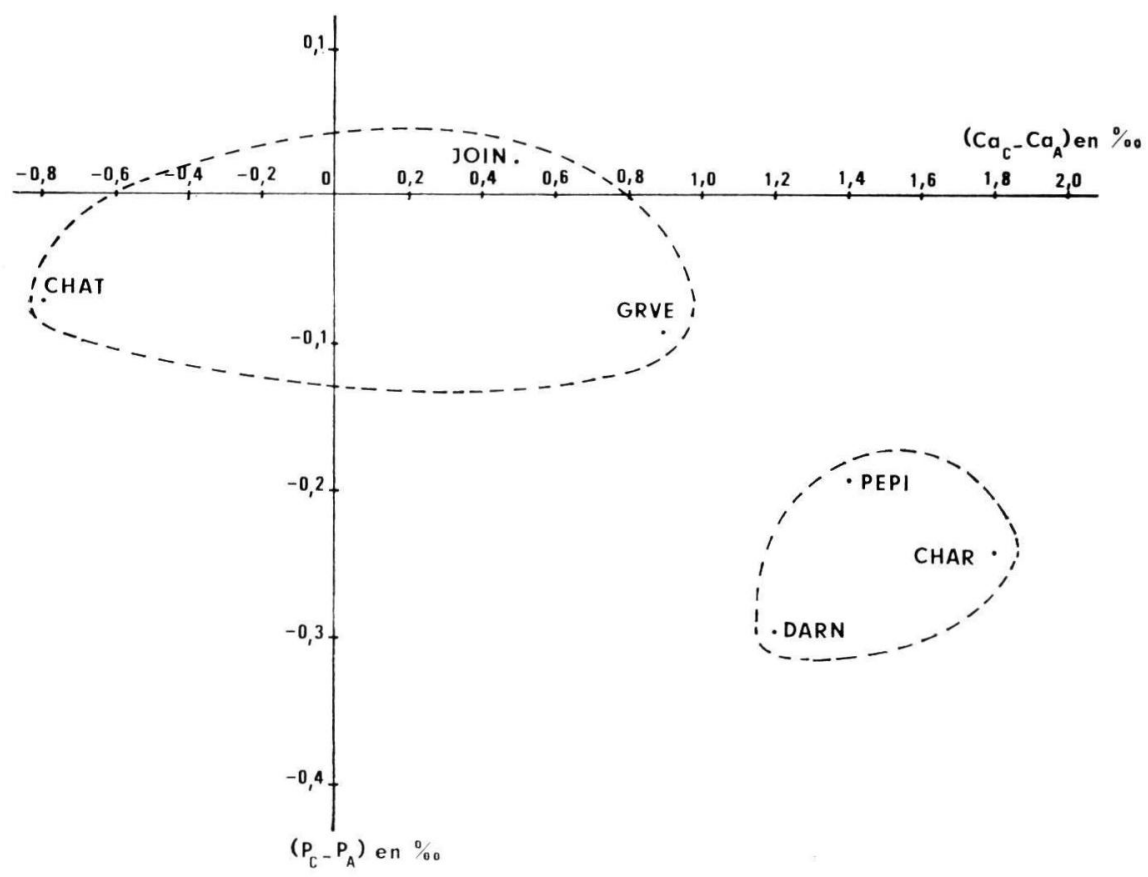

FIG. 11. - Différence d'assimilation du Phosphore en fonction de celle du Calcium, entre le substratum calcaire et le substratum acide.

$\mathrm{Ca}_{\mathrm{c}}=$ concentration en $\mathrm{Ca}$ dans les feuilles en milieu calcaire.

$\mathrm{Ca}_{\mathrm{A}}=$ concentration en $\mathrm{Ca}$ dans les feuilles en milieu acide.

$\mathbf{P}_{\mathbf{c}}=$ concentration en phosphore dans les feuilles en milieu calcaire.

$\mathbf{P}_{\mathrm{A}}=$ concentration en phosphore dans les fevilles en milieu acide.

Difference between alcaline and acide substrates for $\mathrm{P}$ assimilation in leaves in connection with $\mathrm{Ca}$ assimilation.

$\mathrm{Ca}_{\mathrm{c}}=\mathrm{Ca}$ concentration in leaves on alcaline substrate.

$\mathrm{Ca}_{\mathrm{A}}=\mathrm{Ca}$ concentration in leaves on acid substrate.

$\mathrm{P}_{\mathrm{c}}=\mathrm{P}$ concentration in leaves on alcaline substrate.

$P_{A}=P$ concentration in leaves on acid substrate.

sont les provenances calcicoles ou à roche-mère calcaire. Le deuxième groupe a assimilé moins de phosphore et parallèlement plus de calcium, ce sont les provenances de sol acide. Bien entendu, en valeur absolue, les différences entre ces deux groupes restent faibles.

\subsection{Potassium et azote.}

Malgré le choix que nous avons fait et mentionné au paragraphe 6.2, selon lequel nous ne souhaitions pas attribuer une importance trop grande aux valeurs 
absolves des taux d'assimilation des plants, mais plutôt à la modification de ces taux d'un milieu de culture à l'autre, nous devons insister sur le fait que, quel que soit le milieu de culture, le taux d'assimilation du Potassium semble très bas, eu égard aux valeurs normalement rencontrées pour de jeunes plants. Trois hypothèses peuvent être faites :

- le milieu utilisé était carencé en potassium ;

- des semis d'un an n'ont pas une assimilation conforme aux normes généralement admises ;

- le choix des feuilles sur les plants était mauvais. En effet, les feuilles choisies, la paire dominant immédiatement les cotylédons, du fait de leur ancienneté relative sur les plants peuvent avoir un comportement physiologique anormal, se traduisant, par exemple, par une exportation des éléments tels que le połassium vers la tige, plus avancée que des feuilles plus « jeunes », à l'époque du prélèvement, c'est-à-dire début septembre. Quoi qu'il en soit nous ne sommes pas maîtres de cette situation et des phénomènes physiologiques qui peuvent se produire au sein de la plante à un moment donné. Les mettre en cause serait remettre en cause le diagnostic foliaire lui-même. Ce qui importe en l'occurrence est de comparer des échantillons dans des conditions analogues et des variations de teneurs plutôt que des valeurs absolves, tablant sur un parallélisme des phénomènes et une différenciation d'une provenance par rapport à une autre ou d'une même provenance sur deux milieux.

Seule, la première hypothèse est « gênante » pour le devenir des plants. Un milieu carencé en potassium d'après certains auteurs peut perturber profondément la physiologie des semis.

L'analyse chimique des milieux de culture réalisée à la fin de la saison de végétation (tabl. 2) ne porte malheureusement que sur les éléments totaux. Elle ne nous esł donc d'aucune aide pour mettre en évidence une éventuelle carence en potassium échangeable. Le seul élément que nous pouvons apporter pour écarter cette hypothèse de carence est l'aspect «normal » du feuillage des plants qui ne présentait pas une coloration rougeâtre et un dessèchement des bordures des fevilles, comme cela aurait pu être le cas chez des individus carencés en potassium.

A part cela aucune relation ne peut être établie entre l'assimilation de ces deux éléments et celle du calcium. On ne trouve également aucune relation N-P, N-K ou K-P.

\subsection{Conclusions sur le diagnostic foliaire.}

Le principal inconvénient de l'interprétation que nous venons de faire de l'analyse foliaire est qu'elle n'est pas globale. Les éléments ayant été interprétés un par un ou parfois deux par deux en regard des provenances.

Néanmoins, cette démarche se justifie car la meilleure synthèse est faite par les plants eux mêmes qui peuvent précisément réagir à des facteurs nutritionnels limitants simples. Leur croissance est aussi le fruit des synergies et antagonismes de tous ces éléments avec ou sans les caractéristiques génétiques propres à chacune des provenances.

L'hypothèse que nous avons cherché à vérifier est que le développement du hêtre est perturbé lorsque les plants ne sont pas dans un milieu analogue du point 
de vue chimique à celui auquel ils étaient liés sur les stations d'origine. Cela reviendrait donc à vérifier l'hypothèse d'une variabilité d'origine génétique.

S'il y a perturbation, y a-t-il une modification parallèle de l'assimilation minérale ?

Parmi les éléments minéraux analysés, seuls le calcium, le manganèse et le phosphore conduisent à un regroupement des provenances dont l'assimilation concorde :

- d'un côté, des provenances calcicoles ou à roche-mère calcique dont l'assimilation de certains éléments minéraux varie peu avec la concentration en calcium du milieu ( $\mathrm{P}$ et $\mathrm{Ca}$ ), ou dont la variation est peu importante relativement $(\mathrm{Mn})$. Ce sont Chatillon, Joinville et Grand Verneuil ;

- de l'autre, des provenances acidiphiles dont l'assimilation de ces éléments minéraux est perturbée : absorption apparemment " excessive " de calcium en milieu calcaire et carence relative en $\mathrm{Mn}$ et $\mathrm{P}$. Ce sont Darney, Charmes et Petite Pierre.

L'assimilation du Fe, considérée normalement comme un élément déterminant d'un comportement calcifuge, embrouille la situation. Elle range Joinville parmi les provenances acides, ce qui aurait en partie pu être admis, car nous n'avons pas la certitude que l'endroit où le sol a été décrit, correspond bien à la totalité de la zone de récolte des faînes, et, de toutes façons, le pollen provenant d'arbres voisins sur sol limoneux acide peut avoir véhiculé une information propre à une provenance acide (introgression). L'assimilation du fer range aussi Darney parmi les provenances calcicoles ce qui est impensable étant donné la nature de la roche mère : grès à Voltzia ; son $\mathrm{pH}$ et la végétation herbacée : Luzula albida, Polytrichum formosum, Hyperichum pulchrum.

\section{7. - Discussion}

Le hêtre, contrairement à d'autres essences forestières étudiées dans des conditions semblables, n'a pas donné dans nos essais de signes extérieurs frappants de son manque d'adaptation à un milieu auquel il n'est pas habitué. Ce n'est qu'une analyse relativement fine qui permet de mettre en évidence son comportement.

Comme nous l'avons précisé au paragraphe 6.1, nous avons cherché à individualiser des comportements analogues ou très différenciés des provenances, sans jamais essayer de tirer des conclusions sur les valeurs absolues des caractères étudiés. Chacun de ces caractères peut conduire à un classement relatif des provenances (tabl. 5) qui est l'ordre dans lequel elles apparaissent sur les figures de 1 à 11 ou que l'on peut établir grâce aux valeurs du tableau 4.

Le tableau 6, obtenu à partir des rangs de classements rassemblés au tableau 5 met en évidence les points suivants :

- Les trois caractères liés à la croissance et au développement des plants permettent un regroupement : à gauche Joinville, Châtillon et Grand Verneuil, et à droite : Charmes, Petite Pierre et Darney. Ce regroupement est confirmé par les classements des provenances pour les variations d'assimilation de $\mathrm{Ca}, \mathrm{Mn}$ et $\mathrm{P}$. Les présomptions sont donc fortes d'attribuer à ces trois éléments un rôle important dans le changement de comportement des provenances quand on les passe du milieu auquel elles sont habituées à un milieu différent. II est intéressant de noter que ce regroupe- 
TABLEAU 5

Classement des provenances pour chaque caractère

Classification of provenances for each characteristic

\begin{tabular}{|c|c|c|c|c|c|c|}
\hline \multirow{2}{*}{ Caractère } & \multicolumn{6}{|c|}{ Classement } \\
\hline & 1 & 2 & 3 & 4 & 5 & 6 \\
\hline 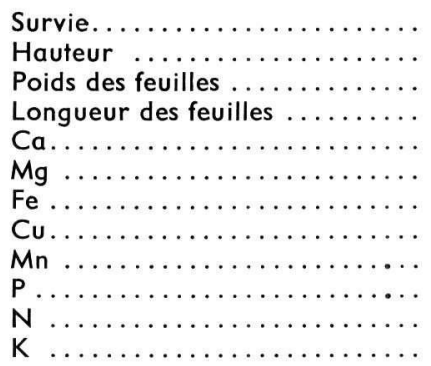 & $\begin{array}{l}\text { PEPI } \\
\text { JOIN } \\
\text { JOIN } \\
\text { CHAT } \\
\text { CHAT } \\
\text { CHAT } \\
\text { CHAT } \\
\text { CHAT } \\
\text { GRVE } \\
\text { JOIN } \\
\text { CHAR } \\
\text { CHAR }\end{array}$ & $\begin{array}{l}\text { CHAT } \\
\text { CHAT } \\
\text { CHAT } \\
\text { JOIN } \\
\text { JOIN } \\
\text { DARN } \\
\text { DARN } \\
\text { CHAR } \\
\text { JOIN } \\
\text { CHAT } \\
\text { GRVE } \\
\text { CHAT }\end{array}$ & $\begin{array}{l}\text { GRVE } \\
\text { GRVE } \\
\text { GRVE } \\
\text { GRVE } \\
\text { GRVE } \\
\text { PEPI } \\
\text { GRVE } \\
\text { GRVE } \\
\text { CHAT } \\
\text { GRVE } \\
\text { CHAT } \\
\text { GRVE }\end{array}$ & $\begin{array}{l}\text { JOIN } \\
\text { CHAR } \\
\text { CHAR } \\
\text { CHAR } \\
\text { DARN } \\
\text { GRVE } \\
\text { CHAR } \\
\text { JOIN } \\
\text { CHAR } \\
\text { PEPI } \\
\text { PEPI } \\
\text { PEPI }\end{array}$ & $\begin{array}{l}\text { CHAR } \\
\text { PEPI } \\
\text { PEPI } \\
\text { PEPI } \\
\text { PEPI } \\
\text { CHAR } \\
\text { JOIN } \\
\text { DARN } \\
\text { PEPI } \\
\text { CHAR } \\
\text { DARN } \\
\text { JOIN }\end{array}$ & $\begin{array}{l}\text { DARN } \\
\text { DARN } \\
\text { DARN } \\
\text { DARN } \\
\text { CHAR } \\
\text { JOIN } \\
\text { PEPI } \\
\text { PEPI } \\
\text { DARN } \\
\text { DARN } \\
\text { JOIN } \\
\text { DARN }\end{array}$ \\
\hline
\end{tabular}

TABLEAU 6

Regroupement des provenances par analogie

Provenances grouped for similarity

\begin{tabular}{|c|c|c|c|c|c|c|}
\hline \multirow{2}{*}{ Caractères } & \multicolumn{6}{|c|}{ Provenances } \\
\hline & Joinville & Châtillon & $\begin{array}{c}\text { Grand } \\
\text { Verneuil }\end{array}$ & Charmes & $\begin{array}{l}\text { Petite } \\
\text { Pierre }\end{array}$ & Darney \\
\hline Hauteur totale .............. & 1 & 2 & 3 & 4 & 5 & 6 \\
\hline Poids des feuilles ............ & 1 & 2 & 3 & 4 & 5 & 6 \\
\hline \multirow[t]{2}{*}{ Longueur des feuilles ......... } & 2 & 1 & 3 & 4 & 5 & 6 \\
\hline & - & - & - & - & - & - \\
\hline 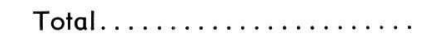 & 4 & 5 & 9 & 12 & 15 & 18 \\
\hline $\mathrm{Ca} \ldots \ldots \ldots \ldots \ldots \ldots \ldots$ & 2 & 1 & 3 & 6 & 5 & 4 \\
\hline 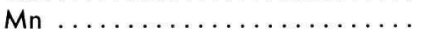 & 2 & 3 & 1 & 4 & 5 & 6 \\
\hline \multirow{2}{*}{$P \ldots \ldots \ldots \ldots \ldots \ldots \ldots$} & 1 & 2 & 3 & 5 & 4 & 6 \\
\hline & - & - & - & - & - & - \\
\hline Total ................ & 5 & 6 & 7 & 15 & 14 & 16 \\
\hline $\mathrm{Fe} \ldots \ldots \ldots \ldots \ldots \ldots$ & 5 & 1 & 3 & 4 & 6 & 2 \\
\hline 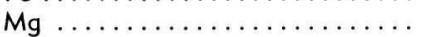 & 6 & 1 & 3 & 5 & 4 & 2 \\
\hline 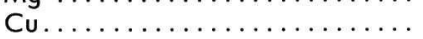 & 4 & 1 & 3 & 2 & 6 & 5 \\
\hline 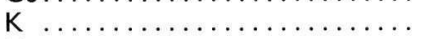 & 5 & 2 & 3 & 1 & 4 & 6 \\
\hline 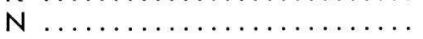 & 6 & $\overline{3}$ & 2 & 1 & 4 & 5 \\
\hline \multirow[t]{2}{*}{ Survie................. } & 4 & 2 & 3 & 5 & 1 & 6 \\
\hline & - & - & - & - & - & - \\
\hline Total. ................. & 30 & 10 & 17 & 18 & 25 & 26 \\
\hline
\end{tabular}

ment consiste précisément à mettre ensemble d'un côté les provenances à sol « calcique » ou à roche-mère calcaire, et de l'autre les provenances à sol acide.

- Les autres caractères étudiés : les éléments $\mathrm{Fe}, \mathrm{Mg}, \mathrm{Cu}, \mathrm{K}$ et $\mathrm{N}$ et la survie, 
conduisent à un classement qui ne diffère du précédent que par le rejet de Joinville dans les provenances à sol acide. Nous avons déjà signalé que cette provenance risquait d'avoir un comportement peu conforme à celui d'une provenance à sol typiquement calcique, du fait de la présence à proximité du lieu supposé de récolte des faines, de parcelles de hêtre dont le sol provient d'un limon éolien plus acide. Ce résultat ne nous surprend donc pas outre mesure.

\section{8. - Conclusion}

Les observations de croissance et les analyses foliaires de semis d'un an de provenances différentes, dont nous venons de relater la teneur, conduisent aux constatations suivantes :

- contrairement à ce qui est dit fréquemment, le hêtre, au niveau spécifique, n'est pas indifférent à la présence de calcaire dans le sol. II faut en effet mettre en évidence sa variabilité intraspécifique pour se rendre compte que différentes provenances de hêtre n'ont pas le même comportement.

Ce comportement variable, dans une expérience de pépinière, n'est dô qu'aux aptitudes génétiques du matériel employé, car toutes les conditions sont identiques hormis les provenances. Une meilleure mycorhization de certaines provenances peut difficilement être invoquée dès la première année, du fait qu'elle n'a pas été réalisée artificiellement ; à moins que précisément elle dépende aussi du matériel génétique.

D'après nos résultats, l'origine du comportement différent des provenances vis-àvis du calcaire pourrait logiquement être recherchée dans les stations dont elles sont issues. On trouve en effet une bonne concordance entre la nature du sol des peuplements d'origine étudiés ef les réactions de nutrition et de croissance des semis qui en sont issus. Des faînes provenant de peuplements sur substrat acide semblent donner naissance à des semis dont la croissance et la nutrition (en dehors de toute consommation de luxe) paraissent meilleures en milieu acide qu'en milieu calcaire. Des semis de provenance "calcaire » se développent mieux en milieu alcalin qu'en milieu acide mais modifient moins leur nutrition que précédemment. En termes génétiques on peut vraisemblablement dire que le milieu a exercé une pression de sélection sur l'espèce dans toutes ses stations d'origine.

Les suggestions pratiques que l'on pourrait tirer de cet essai, si les résultats persistent dans les prochaines années, sont les suivantes :

a) le succès de l'élevage du hêtre en pépinière n'est pas indifférent des provenances utilisées. Une pépinière dont le sol serait calcaire pourrait à un premier stade être moins apte à porter des provenances acides. Par contre, il ne semble pas qu'il y ait d'inconvénient majeur à élever du hêtre "calcaire " en sol acide ;

b) par voie de conséquence, dans la détermination et l'utilisation des Régions de Provenance, il faudra sans doute tenir compte d'une façon plus rigoureuse de la nature du sol ou des éléments floristiques qui permettent de le caractériser. Les fiches de peuplements classés devraient donc comporter ces renseignements. II faudrait aussi proscrire le choix de parcelles à cheval sur des types de sols différents ou proches de parcelles de nature différente (cf. Joinville). 
Les suggestions apportées ci-dessus ef les considérations théoriques dont elles proviennent découlent d'observations faites seulement après une année de croissance depuis le semis. Cette année est primordiale puisqu'elle conditionne le bon développement juvénile, mais l'interprétation du diagnostic foliaire n'est qu'une approche d'une réalité complexe. La poursuite de cette expérience semble donc indispensable, les questions auxquelles nous devrons répondre sont toujours du domaine des interactions sol-provenance. Les caractères étudiés seront principalement les suivants :

- survie après repiquage,

- développement aérien et racinaire,

- assimilation minérale,

- résistance à certaines maladies après inoculation, notamment Nectria coccinea, la cause secondaire de la maladie de l'écorce du hêtre,

- qualité du bois.

Reçu pour publication en novembre 1978.

\section{Summary}

Increment and nutrition of one year old beech seedlings (Fagus silvatica L.) of different provenances, on natural acid substrate and same substrate added with lime.

The question is raised whether the presence of lime in forest soils may exert so much selection pressure on beech populations that transfer of provenances from acid soils to calcareous soils, or vice versa, would cause an abnormal development of plants.

Beechnuts of three provenances from acid soils and of three provenances from calcareous soils or limestone parent material of north-eastern France were pretreated in spring 1977 and sawn on controlled nursery soils. The acid substrate was soil from $A_{1}$ forest horizon ( $\left.\mathrm{pH} 4.9\right)$. The alcaline soil was obtained by adding to the former 5 p. 100 of powdery limestone, raising $\mathrm{pH}$ to 8 .

After one growing season under such conditions, the different provenances vary in reaction towards soils. Those of acid origin develop and grow more poorly on the alcaline substrate than on the acid substrate. This is a consequence of an unbalanced mineral uptake (foliar analysis). On the other hand, alcaline provenances seem to better endure a transfer to an acid soil. Thus, lime in soils may have a toxic action on beech provenances of acid origin. This variability seems to be the consequence of the selecting pressure of the soil in stands.

This study will be continued in the nursery for two years after pricking out and under forest conditions after outplanting.

\section{Références bibliographiques}

CENTRE TECHNIQUE DU BOIS, 1968. Hêtre. Fiches de documentation sur les principales essences des pays tempérés, Paris, $14 \mathrm{P}$.

CENTRE TECHNIQUE DU GÉNIE RURAL DES EAUX ET DES FORÊTS, 1977. Les régions de provenance de hêtre (Fagus silvatica L.). Groupement Technique Forestier. Division « Graines et plants forestiers ». Note Technique, $n^{\circ} 38,33$ p.

GARBAYE J., 1974. Premiers résultats d'un essai de fertilisation de Populus trichocarpa «Fritzi Pauley » sur sol forestier sans nappe dans l'Est de la France. Rev. Forest. Française, 26 (5), 379-387.

GARBAYE J., LE TACON F., 1974. Ełude expérimentale du comportement de divers clones de résineux ef de deux clones de peupliers vis-à-vis du pH et de la teneur en calcaire du sol. Rev. Forest. Française, 26 (6), 439-446.

KELLER R., LE TACON, TIMBAL J., 1976. La densité du bois de hêtre dans le nord-est de la France. Influence des caractéristiques du milieu et du type de sylviculture. Ann. Sci. Forest., 33 (1), 1-17.

LE TACON F., NYS C., 1970. Les sols du massif de Haye et leur influence sur le comportement des réserves de hêtre en taillis sous futaie. Rev. Forest. Française, 22 (5), 545-552. 
LE TACON F., TIMBAL J., 1973. Valeurs indicatrices des principales espèces végétales des hêtraie du nord-est de la France, vis-à-vis des types d'humus. Rev. Forest. Française, 25 (4), 269-282.

LE TACON F., TOUTAIN F., 1973. Variations saisonnières et stationnelles de la teneur en éléments minéraux des feuilles de hêtre (Fagus silvatica) dans l'est de la France. Ann. Sci. Forest., 30 (1), 1-29.

POLGE H., KELLER R., THIERCELIN F., 1972. Effet du sol et de l'hérédité sur la croissance et les caractéristiques anatomiques de jeunes plants de hêtre. C.N.R.F. Nancy, Station de Recherches sur la Qualité des Bois. Doc. no 1, 24 p.

TIMBAL J., 1974. Principaux caractères écologiques et floristiques des hêtraies du nord-est de la France. Ann. Sci. Forest., 31 (1), 27-45. 